\title{
A Josephson quantum electron pump
}

\author{
Francesco Giazotto ${ }^{1 \star}$, Panayotis Spathis ${ }^{1}$, Stefano Roddaro ${ }^{1}$, Subhajit Biswas ${ }^{1}$, Fabio Taddei ${ }^{1}$, \\ Michele Governale ${ }^{2}$ and Lucia Sorba ${ }^{1}$
}

\begin{abstract}
Mesoscopic charge pumping, a transport mechanism that relies on the explicit time-dependence of some properties of a nanoscale conductor, was envisaged theoretically a few decades $\mathrm{ago}^{1-4}$. So far, nanoscale pumps have been realized only in systems exhibiting strong Coulombic effects ${ }^{5-12}$, whereas evidence for pumping in the absence of Coulomb blockade has been elusive. A pioneering experiment by Switkes et al. $^{13}$ evidenced the difficulty of modulating in time the properties of an open mesoscopic conductor at cryogenic temperatures without generating undesired bias voltages due to stray capacitances ${ }^{14,15}$. One possible solution to this problem is to use the a.c. Josephson effect to induce periodically timedependent Andreev reflection amplitudes in a hybrid normalsuperconducting system ${ }^{16}$. Here we report the experimental detection of charge flow in an unbiased InAs nanowire embedded in a superconducting quantum interference device (SQUID). In this system, quantum pumping may occur via the cyclic modulation of the phase of the order parameter of different superconducting electrodes. The symmetry of the current with respect to the enclosed magnetic flux ${ }^{17,18}$ and bias SQUID current is a discriminating signature of pumping. Currents exceeding $20 \mathrm{pA}$ are measured at $250 \mathrm{mK}$, and exhibit symmetries compatible with quantum pumping.
\end{abstract}

The microscopic mechanism that enables the transport properties of the NW to be affected by the phases of the superconducting order parameter is Andreev reflection ${ }^{19}$. This is the quantum process by which an electron impinging from the normal side onto the interface between a normal metal and a superconductor is retroreflected as a hole (that is, a time-reversed electron) that picks up the phase of the superconducting order parameter. When two or more superconductors are connected to the nanowire (NW), multiple Andreev scattering processes can occur between them such that transport through the NW will depend on the differences between the phases of the order parameters ${ }^{20}$.

The physical realization of this scheme is shown in Fig. 1a and consists of a heavily-doped InAs semiconducting NW on top of which three fingers of superconducting $(\mathrm{S})$ vanadium $(\mathrm{V})$ are deposited, thus implementing a superconducting quantum interference device (SQUID, ref. 21). Two Au normal-metal electrodes $(\mathrm{N})$ are coupled to the ends of the NW to allow detection of the current $I_{\text {wire }}$ flowing through the wire. A close-up of the device core is shown in Fig. 1b. Time-dependence, and possibly pumping, arises from biasing the loop with a current $I_{S Q U I D}$ larger than the critical current $I_{\mathrm{c}}$ of the SQUID, thus the phase differences $\varphi_{1}(t)$ and $\varphi_{2}(t)$ across the two Josephson junctions cycle in time at the Josephson frequency $v_{\mathrm{J}}=V_{\text {SQUID }} / \Phi_{0}$, where $V_{\text {SQUID }}$ is the voltage developed across the SQUID and $\Phi_{0} \simeq 2 \times 10^{-15} \mathrm{~Wb}$ is the flux quantum. Furthermore, $\varphi_{1}(t)$ and $\varphi_{2}(t)$ can be shifted
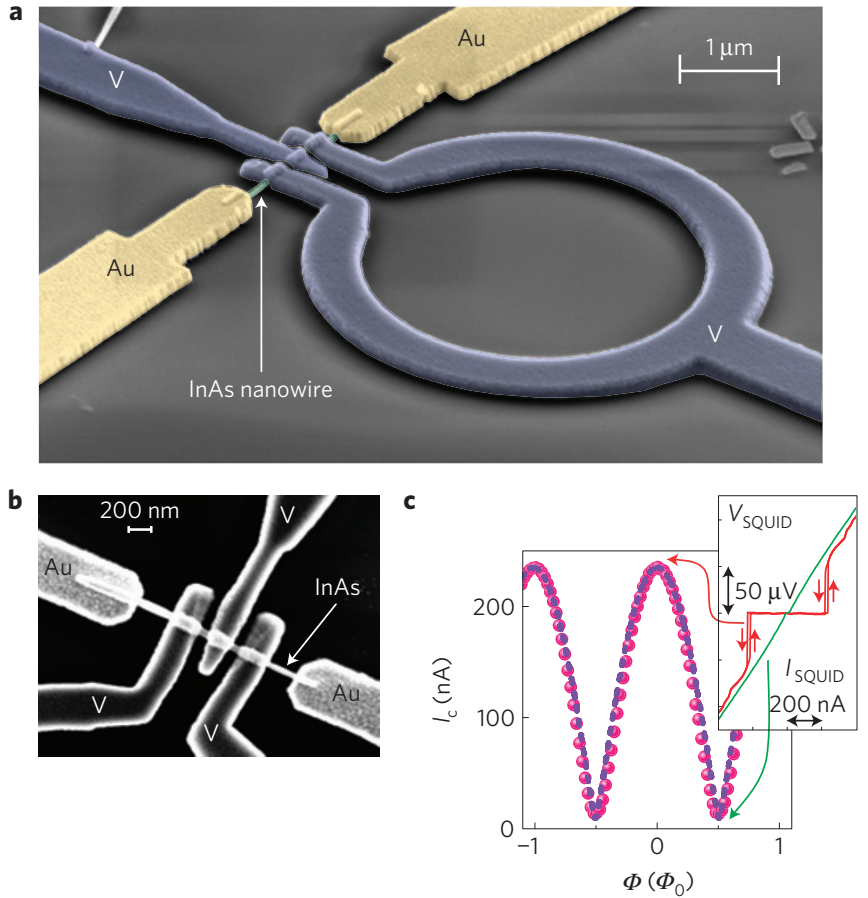

Figure 1 | InAs Josephson quantum electron pump. a, Pseudo-colour scanning electron micrograph of the device. An InAs nanowire (NW) is connected to three $\simeq 250$-nm-wide $\mathrm{V} / \mathrm{Ti}$ superconducting contacts, forming two $\simeq 50$-nm-long Josephson weak-links and realizing a superconducting quantum interference device (SQUID). Two Au/Ti leads, placed at a relative distance of $\simeq 1.5 \mu \mathrm{m}$, are contacted to the ends of the NW to allow current detection. The structure was fabricated using electron-beam lithography and evaporation of metals. The normal-state resistance of the SQUID is $\sim 250 \Omega$, whereas that of the Au/NW/Au line is $\sim 3.5 \mathrm{k} \Omega$. b. Blow-up of the device core showing the two $\mathrm{V} / \mathrm{In} \mathrm{As} / \mathrm{V}$ Josephson junctions and the two Au electrodes. c, Inset: SQUID voltage ( $V_{\text {SQUID }}$ ) versus current (/SQUID) characteristics at $\Phi=0$ and $\Phi=\Phi_{0} / 2$ ( $\Phi$ is the applied magnetic flux and $\Phi_{0}$ is the flux quantum) showing a maximum critical current of $\sim 235 \mathrm{nA}$. $\Phi_{0}$ corresponds to a magnetic field of $\simeq 1.4$ Oe applied through an effective loop area of $\sim 14.6 \mu \mathrm{m}^{2}$. Main panel: $\Phi$-dependent modulation of the SQUID critical current $I_{c}$. Dashed line is the theoretical behaviour of a tunnel and resistively-shunted junction SQUID assuming an asymmetry of $~ 4 \%$ between the critical currents of the two weak-links. Data in c are taken at $T=250 \mathrm{mK}$.

by a constant term $\delta \varphi=2 \pi \Phi / \Phi_{0}$ originating from an applied magnetic flux $\Phi$ threading the loop. This scheme has the advantage

${ }^{1}$ NEST, Istituto Nanoscienze-CNR and Scuola Normale Superiore, Piazza S. Silvestro 12, I-56127 Pisa, Italy, ${ }^{2}$ School of Chemical and Physical Sciences and MacDiarmid Institute for Advanced Materials and Nanotechnology, Victoria University of Wellington, P.O. Box 600, Wellington 6140, New Zealand. *e-mail: giazotto@sns.it. 

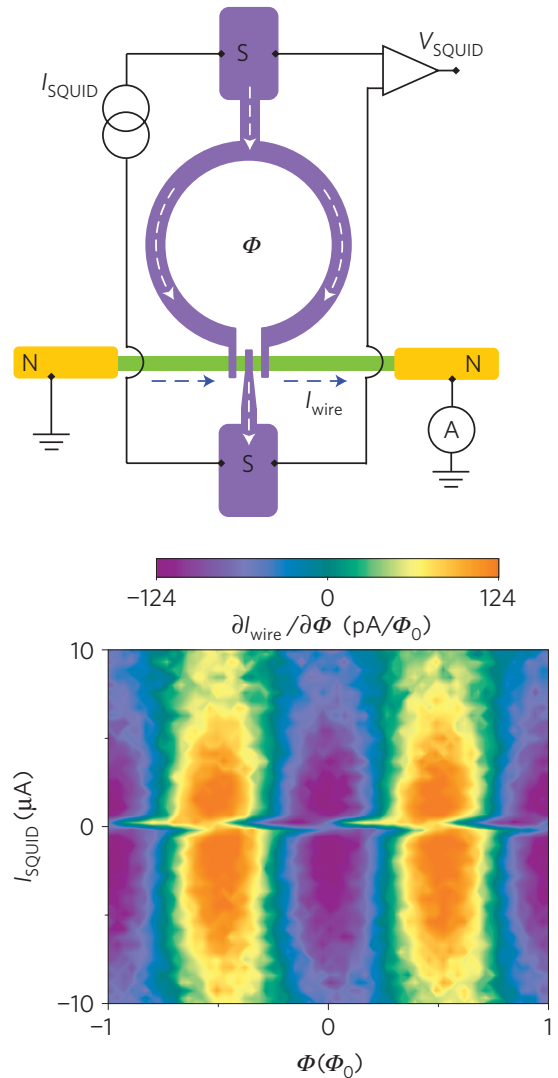

b

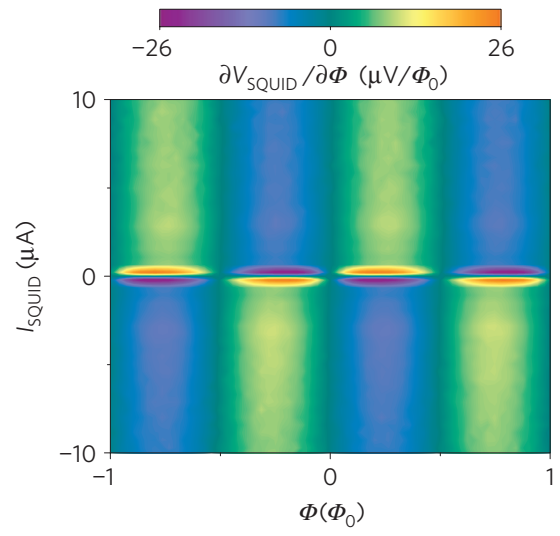

d

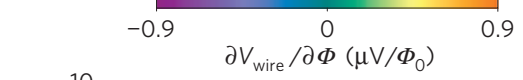

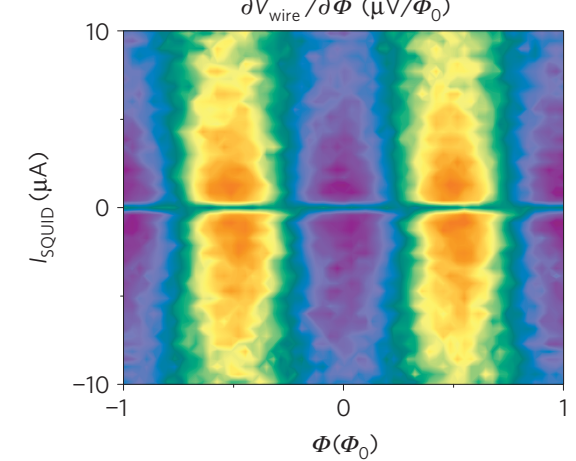

Figure 2 | Experiment set-up and transfer function characteristics. a, Schematic drawing of the Josephson quantum electron pump set-up. A d.c. current $I_{\text {SQUID }}$ is fed into the SQUID terminals through a floating source while the voltage drop $V_{\text {SQUID }}$ is recorded against the applied magnetic flux $\Phi$ threading the ring. When $I_{S Q U I D}$ exceeds the SQUID critical supercurrent the a.c. Josephson effect sets in, inducing a current $I_{\text {wire }}$ which flows in the NW. $I_{\text {wire }}$ is sensed through an ammeter. S and N denote superconductors and normal metals, respectively. $\mathbf{b}$, Colour plot of the SQUID flux-to-voltage transfer function $\mathcal{V}_{\text {SQUID }}=\partial V_{\text {SQUID }} / \partial \Phi$ versus $\Phi$ and $I_{\text {SQUID }} . \mathcal{V}_{\text {SQUID }}$ is antisymmetric in $\Phi$ and $I_{\text {SQUID }}$. c, Colour plot of the NW flux-to-current transfer function $\mathcal{I}_{\text {wire }}=\partial I_{\text {wire }} / \partial \Phi$ versus $\Phi$ and $I_{\text {SQUID }} \cdot \mathbf{d}$, Colour plot of the NW flux-to-voltage transfer function $\mathcal{V}_{\text {wire }}=\partial V_{\text {wire }} / \partial \Phi$ versus $\Phi$ and $I_{S Q U I D}$. Data are taken with a voltmeter in an open-circuit configuration, that is, without allowing $I_{\text {wire }}$ to flow. Note the markedly different behaviour exhibited by $\mathcal{I}_{\text {wire }}$ and $\mathcal{V}_{\text {wire, }}$ which are almost symmetric in $\Phi$ as well as in ISQUID. All measurements are taken at $T=250 \mathrm{mK}$ using a low-frequency phase-sensitive technique to get higher sensitivity and reduced noise.

that no high-frequency signal needs to be applied to the sample, thus simplifying the set-up and minimizing the impact of stray capacitances: the time-dependent signal is self-generated as a result of the a.c. Josephson effect.

Below the critical temperature of the superconductors $\left(T_{\mathrm{c}} \simeq 4.65 \mathrm{~K}\right)$ a Josephson current flows through the SQUID across the NW. The SQUID voltage-current characteristics at $250 \mathrm{mK}$ are shown in the inset of Fig. 1c for two representative values of $\Phi$. Whereas for $\Phi=0$ the characteristic shows a clear dissipationless regime with a critical current $I_{\mathrm{c}} \simeq 235 \mathrm{nA}$, for $\Phi=\Phi_{0} / 2$ it behaves almost linearly, with $I_{\mathrm{c}}$ largely suppressed. The full $I_{\mathrm{c}}(\Phi)$ dependence (main panel of Fig. 1c) shows the characteristic pattern of a superconducting interferometer. The theoretical curve of a conventional (that is, described by the RSJ model) SQUID (ref. 22) is shown for comparison (dashed line, see Supplementary Information).

Figure 2a shows a sketch of the pumping measurement set-up. A d.c. current $I_{\text {SQUID }}$ is fed through the SQUID terminals while the voltage drop $V_{\text {SQUID }}$ is measured against $\Phi$. The $\mathrm{N}$ electrodes are grounded and $I_{\text {wire }}$ is sensed with an ammeter. The $\mathrm{N}$ and $\mathrm{S}$ parts of the circuit have no common ground, therefore preventing any direct net charge transfer from the SQUID to the NW.

In the following we will concentrate our attention on the symmetries in $\Phi$ and $I_{\text {SQUID }}$ exhibited by the measured signal, as these are of crucial importance for the interpretation of the experiment. The low-temperature SQUID flux-to-voltage transfer function $\mathcal{V}_{\text {SQUID }}=\partial V_{\text {SQUID }} / \partial \Phi$ versus $\Phi$ and $I_{\text {SQUID }}$ is shown in Fig. 2b. In particular, $\mathcal{V}_{\text {SQUID }}$ is a $\Phi_{0}$-periodic function of $\Phi$ and is antisymmetric in $\Phi$ and $I_{\text {SQUID }}$. By contrast, the flux-to-current transfer function of the NW, $\mathcal{I}_{\text {wire }}=\partial I_{\text {wire }} / \partial \Phi$ (Fig. 2c), besides exhibiting the same periodicity in $\Phi_{0}$, shows a drastically different behaviour, being almost symmetric in both $\Phi$ and $I_{\text {SQUID }}$. A similar behaviour, with the same symmetries of $\mathcal{I}_{\text {wire }}$, is exhibited by the $\mathrm{NW}$ flux-to-voltage transfer function, $\mathcal{V}_{\text {wire }}=\partial V_{\text {wire }} / \partial \Phi$ (Fig. $2 \mathrm{~d}$ ), where $V_{\text {wire }}$ is measured with open NW contacts. $\mathcal{I}_{\text {wire }}$ and $\mathcal{V}_{\text {wire }}$ result from different but complementary measurements, and the evidence of such a similarity suggests that both represent the same physical mechanism (see Supplementary Information). As we shall argue, the nature of the symmetries exhibited by $\mathcal{I}_{\text {wire }}$ and $\mathcal{V}_{\text {wire }}$ is compatible with quantum pumping mechanisms.

In general, the pumped current is not expected to show definite parity with $\Phi$ (refs 17,18 ), therefore $I_{\text {wire }}$ can have a flux-symmetric component as well. This, however, could be ascribed also to other mechanisms than pumping. In addition, $I_{\text {wire }}$ is not even expected to possess any definite parity with $I_{\text {SQUID }}$. To extract a pure pumped current contribution from the whole measured signal, we focus on the component of $I_{\text {wire }}$ that is antisymmetric in $\Phi, I_{\text {wire }}^{\mathrm{A}}$, as it is predicted to be a fingerprint of quantum pumping in the Josephson quantum electron pump (JQEP, ref. 16). After $\Phi$-integration of $\mathcal{I}_{\text {wire }}, I_{\text {wire }}^{\mathrm{A}}$ is obtained as $I_{\text {wire }}^{\mathrm{A}}=\left[I_{\text {wire }}\left(\Phi, I_{\text {SQUID }}\right)-I_{\text {wire }}\left(-\Phi, I_{\text {SQUID }}\right)\right] / 2$. The result of this procedure is shown in Fig. 3a, which shows $I_{\text {wire }}^{\mathrm{A}}$ 

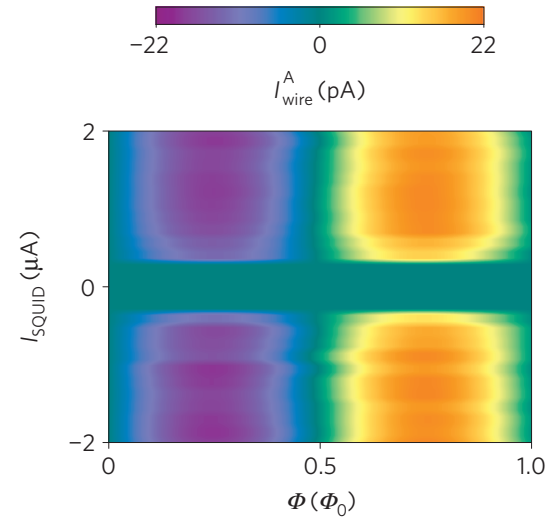

c
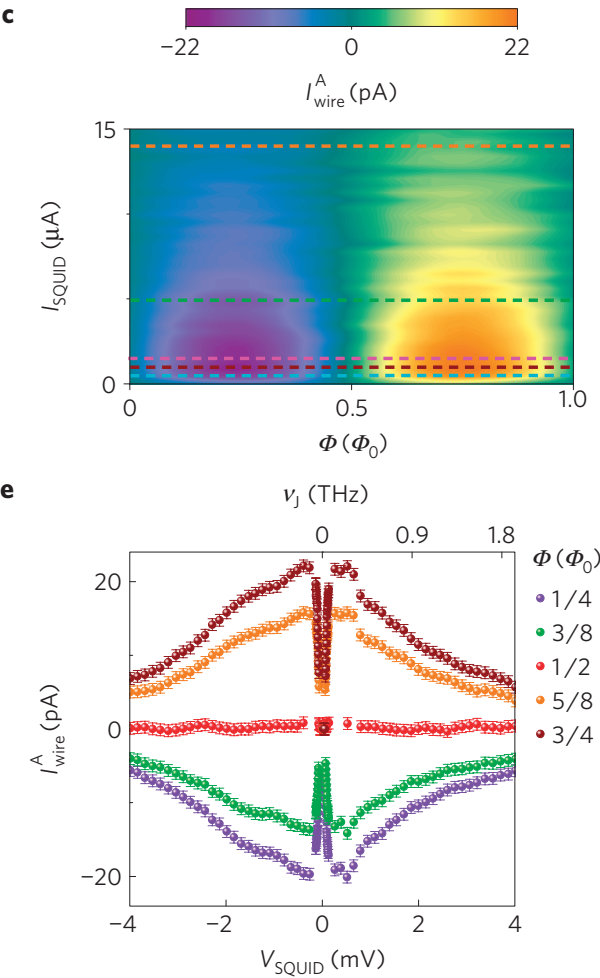
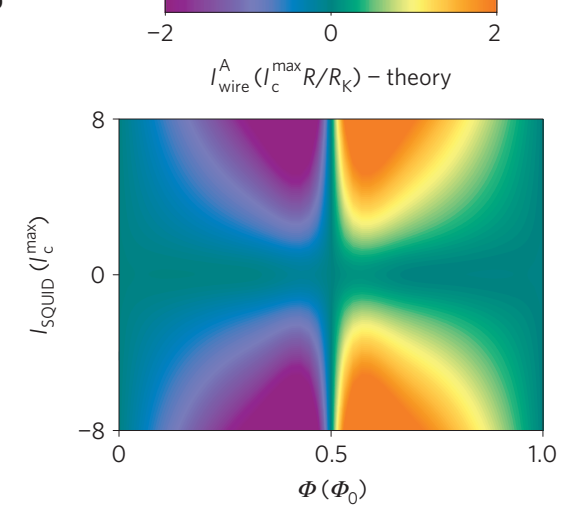

d

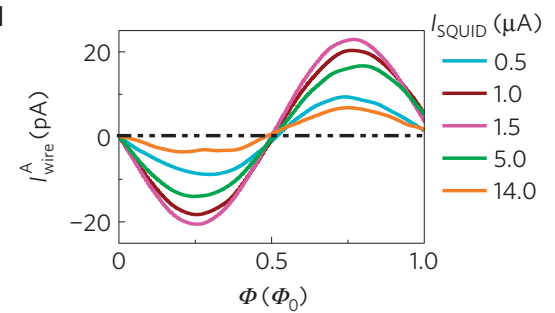

f

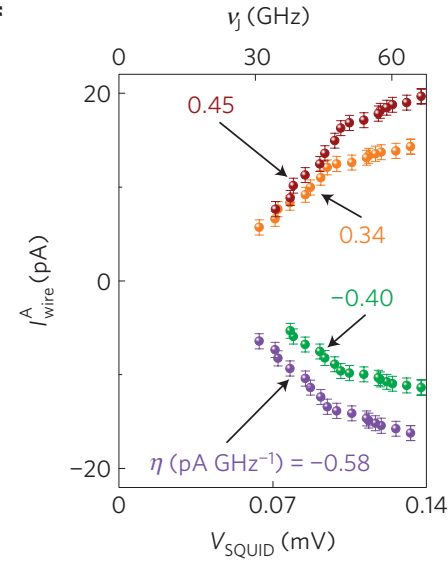

Figure 3 | Flux and $I_{\text {SQUID }}$ dependence of the antisymmetric part of the current flowing in the NW. a, Colour plot of $I_{\text {wire }}^{A}$ versus $I_{\text {SQUID }}$ and $\Phi$. $\mathbf{b}$, Colour plot of the theoretical zero-temperature $I_{\text {wire }}^{A}$ versus ISQUID and $\Phi$. The calculation was performed for the JQEP geometry, assuming the same asymmetry

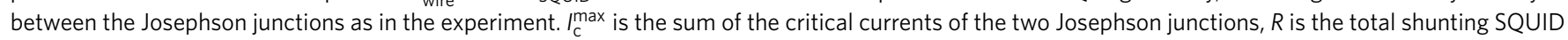

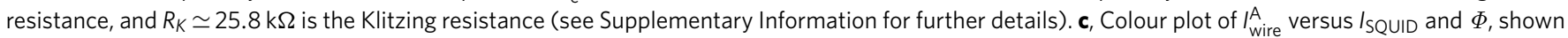
over a wider range of $I_{\text {SQUID. }} \mathbf{d}$, $I_{\text {wire }}^{\mathrm{A}}$ versus $\Phi$ for a few representative values of $I_{\text {SQUID. }}$. The latter are indicated as dashed lines of the same colour as in $\mathbf{c}$.

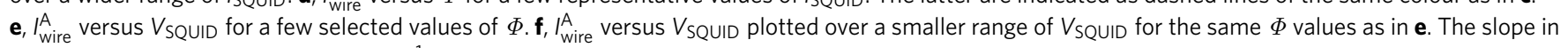
the linear regime, expressed in $\mathrm{pA} \mathrm{GHz}{ }^{-1}$, is denoted as $\eta$. In $\mathbf{e}$ and $\mathbf{f}$ the error bars represent the standard deviation of the current values calculated over several measurements, and the upper horizontal scale is expressed in terms of the Josephson frequency $v_{\mathrm{J}}$. All measurements are taken at $T=250$ mK.

versus $\Phi$ and $I_{\text {SQUID }}$ at $250 \mathrm{mK}$. The $\Phi_{0}$ periodicity together with the antisymmetry imply that $I_{\text {wire }}^{\mathrm{A}}$ vanishes at $\Phi=\Phi_{0} / 2$, and its sign and magnitude can be changed by varying $\Phi$. Notably, $I_{\text {wire }}^{\mathrm{A}}$ is almost symmetric in $I_{\text {SQUID }}$. The theoretical $I_{\text {wire }}^{\mathrm{A}}$ calculated for the JQEP geometry through a dynamical scattering approach ${ }^{23-25}$, assuming the existence of multiple independent modes in the NW, is shown in Fig. 3b (see Supplementary Information). Although rather idealized, the model is an essential tool to predict the pumped current symmetries of the JQEP. Remarkably, summing over many NW modes yields $I_{\text {wire }}^{\mathrm{A}}$, which is almost symmetric in $I_{\mathrm{SQUID}}$, in agreement with the experiment.

Figure $3 \mathrm{c}$ shows $I_{\text {wire }}^{\mathrm{A}}$ versus $\Phi$ and $I_{\mathrm{SQUID}}$ over a wider range of SQUID currents. Specifically, $I_{\text {wire }}^{\mathrm{A}}$ turns out to be a non-monotonic function of $I_{\text {SQUID }}$, initially increasing, then being suppressed for large $I_{\text {SQUID }}$. This is emphasized in Fig. 3d, where $I_{\text {wire }}^{\mathrm{A}}(\Phi)$ is plotted for selected values of $I_{\text {SQUID }} . I_{\text {wire }}^{\mathrm{A}}$ is a sinusoidal-like function of $\Phi$, with an amplitude that depends on $I_{\mathrm{SQUID}}$, and reaches its maximum at $\Phi \sim(1 / 4) \Phi_{0}$ and $\Phi \sim(3 / 4) \Phi_{0}$.

The full $I_{\text {wire }}^{\mathrm{A}}\left(V_{\text {SQUID }}\right)$ dependence for a few values of flux is shown in Fig. 3e and highlights both the monotonic linear increase for low $V_{\text {SQUID }}$ and suppression at large $V_{\text {SQUID }}$. The symmetry in $V_{\text {SQUID }}$ (that is, in $I_{\text {SQUID }}$ ) is emphasized as well. Furthermore, $\left|I_{\text {wire }}^{\mathrm{A}}\right|$ is maximized at $\left|V_{\text {SQUID }}^{\max }\right| \approx 0.4 \mathrm{mV}$, independently of $\Phi$, where it reaches values exceeding $20 \mathrm{pA}$. By converting $V_{\text {SQUID }}^{\max }$ in terms of the Josephson frequency we get $\nu_{\mathrm{J}} \simeq 190 \mathrm{GHz}$, with a corresponding time, $v_{\mathrm{J}}^{-1} \sim 5 \mathrm{ps}$, which is comparable to $\tau_{D}=W^{2} / D \simeq 4 \mathrm{ps}$, that is, the time required by electrons to diffuse in the $\mathrm{NW}$ between the Josephson junctions. In the above expression $W \simeq 250 \mathrm{~nm}$ 


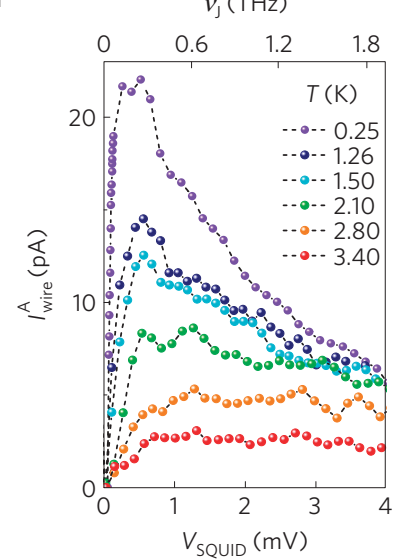

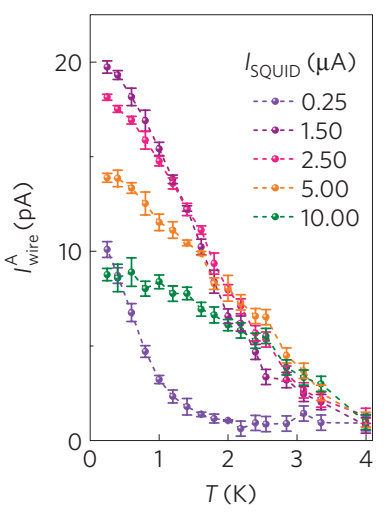

Figure 4 | Temperature dependence of the antisymmetric part of the current flowing in the NW. a, $I_{\text {wire }}^{\mathrm{A}}$ versus I SQUID $_{\text {measured at several bath }}$ temperatures $T$. b, $I_{\text {wire }}^{\mathrm{A}}$ versus $T$ at selected bias currents $I_{\text {SQUID. Note the }}$ saturation of $I_{\text {wire }}^{\mathrm{A}}$ at low temperature as well as its suppression at high temperature. The error bars represent the standard deviation of the current values calculated over several measurements. Dashed lines in both $\mathbf{a}$ and $\mathbf{b}$ are guides to the eye, and all measurements are taken for $\Phi=(3 / 4) \Phi_{0}$.

is the width of the SQUID central electrode (Fig. 1b), which we assume to coincide with the separation between the weak-links, whereas $D \simeq 0.015 \mathrm{~m}^{2} \mathrm{~s}^{-1}$ is the diffusion coefficient of the NW (ref. 26). The transition between the regime of $I_{\text {wire }}^{\mathrm{A}}$ enhancement as a function of $V_{\text {SQUID }}$ to the one of $I_{\text {wire }}^{\mathrm{A}}$ suppression can be explained in terms of the ability of the electrons to follow adiabatically the time-dependent parameters up to a maximum frequency set by $\tau_{D}^{-1}$. Another possible contribution to the suppression observed at higher $V_{\text {SQUID }}$ might stem from weakening of the a.c. Josephson coupling at high applied current ${ }^{27}$.

The $I_{\text {wire }}^{\mathrm{A}}\left(V_{\text {SQUID }}\right)$ dependence, plotted over a reduced bias range, is shown in Fig. 3f. In particular, $I_{\text {wire }}^{\mathrm{A}}$ shows a linear behaviour, with a slope $\eta$ that depends on the applied flux and achieves values as high as several $10^{-1} \mathrm{pA} \mathrm{GHz}^{-1}$. In the so-called 'adiabatic regime', that is, where the pumped current is expected to vary linearly with frequency, $\eta$ would therefore correspond to some $10^{-3}$ electrons per pump cycle.

The role of temperature $(T)$ is shown in Fig. $4 \mathrm{a}$, which shows $I_{\text {wire }}^{\mathrm{A}}$ versus $V_{\text {SQUID }}$ at $\Phi=(3 / 4) \Phi_{0}$ for increasing temperatures. $I_{\text {wire }}^{\mathrm{A}}$ monotonically decreases on increasing $T$, which can be ascribed to the influence of thermal smearing as well as thermally induced dephasing, and is suppressed for $T \gtrsim 3.5 \mathrm{~K}$. We stress that the aforementioned temperature is substantially smaller than $T_{\mathrm{c}}$, the latter setting the disappearance of both the Josephson effect and superconductivity in the JQEP. The $I_{\text {wire }}^{\mathrm{A}}(T)$ dependence at the same flux is shown in Fig. $4 \mathrm{~b}$ for a few $I_{\text {SQUID }}$ values. Specifically, $I_{\text {wire }}^{\mathrm{A}}$ begins to round off at lower temperatures, indicating a saturation, whereas it is damped at higher $T$. The low-temperature behaviour suggests that current tends to saturate on reducing temperature when the 'effective' separation between Josephson junctions becomes of the same order as the electron thermal coherence length in the NW, $L_{\mathrm{T}}=\sqrt{\hbar D /\left(2 \pi k_{\mathrm{B}} T\right)} \sim 270 \mathrm{~nm}$ at $250 \mathrm{mK}$, where $\hbar$ is the reduced Planck constant and $k_{\mathrm{B}}$ is the Boltzmann constant. By contrast, the decay of $L_{\mathrm{T}}$ at higher temperatures may be considered as one of the predominant decoherence mechanisms leading to $I_{\text {wire }}^{\mathrm{A}}$ suppression. Further study is needed to clarify this point.

It is worthwhile emphasizing that other effects that might manifest in the JQEP would yield currents characterized by symmetries markedly different from the ones predicted for quantum pumping (see Supplementary Information). Among these

we note (1) any spurious current due to asymmetry between the junctions, which is always dominated by a component symmetric in $\Phi$ and antisymmetric in $I_{\text {SQUID }} ;(2)$ any thermocurrent generated by a different power dissipated in the two junctions, which is expected to be predominantly symmetric in both $\Phi$ and $I_{\text {SQUID }}$.

We finally note that other normal conductors than InAs NWs could be used for the implementation of the JQEP. This might pave the way for the investigation of the interplay between superconductivity-induced quantum pumping and exotic electronic states existing, for instance, in graphene ${ }^{28}$ or in carbon nanotubes ${ }^{29}$.

\section{Methods}

Selenium-doped InAs NWs were grown by chemical beam epitaxy on an InAs $111 \mathrm{~B}$ substrate. Gold catalyst particles were formed by thermal dewetting (at $520^{\circ} \mathrm{C}$ for $20 \mathrm{~min}$ ) of a 0.5 -nm-thick Au film under tertiarybutylarsine (TBA) flux. NWs were grown for $2 \mathrm{~h}$ at $420^{\circ} \mathrm{C}$ using TBA, trimethylindium (TMI) and ditertiarybutylselenide (DTBSe) metallorganic precursors with line pressures of 2.0 torr, 0.3 torr, and 0.4 torr, respectively. NWs have diameters of $90 \pm 10 \mathrm{~nm}$ and are around $2.5 \mu \mathrm{m}$ long. Transport parameters were estimated over an ensemble of nominally identical $1 \mu \mathrm{m}$-long NW field effect transistors using a charge control model ${ }^{30}$ and a numerical evaluation of the gate capacitance. Carrier density was estimated to be $n=1.8 \pm 0.8 \times 10^{19} \mathrm{~cm}^{-3}$ and electron mobility $\mu=300 \pm 100 \mathrm{~cm}^{2} \mathrm{~V}^{-1} \mathrm{~s}^{-1}$. The devices were fabricated using a technique of dry cleavage of the NWs onto $\mathrm{Si} / \mathrm{SiO}_{2}$ substrates $(500 \mathrm{~nm}$ oxide on intrinsic $\mathrm{Si}$ ). Contacts were obtained by a two-step aligned process: thermal evaporation of Ti/Au $(10 / 80 \mathrm{~nm})$ was performed first and followed by electron-beam deposition of $\mathrm{Ti} / \mathrm{V}$ $(15 / 120 \mathrm{~nm})$ in a UHV chamber ${ }^{21}$. InAs NWs were treated with a $\mathrm{NH}_{4} \mathrm{~S}_{x}$ solution before each evaporation step to obtain transparent metal-NW contacts ${ }^{26}$.

The magneto-electric characterization of the devices was performed in a filtered ${ }^{3} \mathrm{He}$ refrigerator (two-stage RC- and $\pi$-filters) down to $\sim 250 \mathrm{mK}$ using a standard 4-wire technique. Current injection at the SQUID terminals was obtained by using a battery-powered floating source, with voltage and current being measured by room-temperature preamplifiers. Derivative measurements (flux-to-voltage as well as flux-to-current transfer functions) were performed with a standard low-frequency lock-in technique by superimposing a small modulation on the applied magnetic field.

Received 22 February 2011; accepted 22 June 2011; published online 31 July 2011

\section{References}

1. Thouless, D. J. Quantization of particle transport. Phys. Rev. B 27, 6083-6087 (1983).

2. Büttiker, M., Thomas, H. \& Prêtre, A. Current partition in multiprobe conductors in the presence of slowly oscillating external potentials. Z. Phys. B 94, 133-137 (1994).

3. Brouwer, P. W. Scattering approach to parametric pumping. Phys. Rev. B 58, R10135-R10138 (1998).

4. Zhou, F., Spivak, B. \& and Altshuler, B. Mesoscopic mechanism of adiabatic charge transport. Phys. Rev. Lett. 82, 608-611 (1999).

5. Pothier, H., Lafarge, P., Urbina, C., Esteve, D. \& Devoret, M. H. Single-electron pump based on charging effects. Europhys. Lett. 17, 249-254 (1992).

6. Martinis, J. M., Nahum, M. \& Jensen, H. D. Metrological accuracy of the electron pump. Phys. Rev. Lett. 72, 904-907 (1994).

7. Fletcher, N. E. et al. Quantized acoustoelectric current transport through a static quantum dot using a surface acoustic wave. Phys. Rev. B 68, 245310 (2003).

8. Ebbecke, J. et al. Quantized charge pumping through a quantum dot by surface acoustic waves. Appl. Phys. Lett. 84, 4319-4321 (2004).

9. Pekola, J. P. et al. Hybrid single-electron transistor as a source of quantized electric current. Nature Phys. 4, 120-124 (2008).

10. Fuhrer, A., Fasth, C. \& Samuelson, L. Single electron pumping in InAs nanowire double quantum dots. Appl. Phys. Lett. 91, 052109 (2007).

11. Buitelaar, M. R. et al. Adiabatic charge pumping in carbon nanotube quantum dots. Phys. Rev. Lett. 101, 126803 (2008).

12. Kaestner, B. et al. Robust single-parameter quantized charge pumping. Appl. Phys. Lett. 92, 192106 (2008).

13. Switkes, M., Marcus, C. M., Campman, K. \& Gossard, A. C. An adiabatic quantum electron pump. Science 283, 1905-1908 (1999).

14. Brouwer, P. W. Rectification of displacement currents in an adiabatic electron pump. Phys. Rev. B 63, 121303(R) (2001).

15. DiCarlo, L., Marcus, C. M. \& Harris, J. S. Jr Photocurrent, rectification, and magnetic field symmetry of induced current through quantum dots. Phys. Rev. Lett. 91, 246804 (2003).

16. Russo, S., Tobiska, J., Klapwijk, T. M. \& Morpurgo, A. F. Adiabatic quantum pumping at the Josephson frequency. Phys. Rev. Lett. 99, 086601 (2007). 
17. Shutenko, T. A., Altshuler, B. L. \& Aleiner, I. L. Mesoscopic fluctuations of adiabatic charge pumping in quantum dots. Phys. Rev. B 61, 10366-10375 (2000).

18. Moskalets, M. \& Büttiker, M. Magnetic-field symmetry of pump currents of adiabatically driven mesoscopic structures. Phys. Rev. B 72, 035324 (2005).

19. Andreev, A. F. Thermal conductivity of the intermediate state of superconductors. Sov. Phys. JETP 19, 1228-1231 (1964).

20. Giazotto, F., Peltonen, J. T., Meschke, M. \& Pekola, J. P. Superconducting quantum interference proximity transistor. Nature Phys. 6, 254-259 (2010).

21. Spathis, P. et al. Hybrid InAs nanowire-vanadium proximity SQUID. Nanotechnology 22, 105201 (2011).

22. Tinkham, M. Introduction to Superconductivity 2nd edn (McGraw-Hill, Inc., 1996).

23. Wang, J., Wei, Y., Wang, B. \& Guo, H. Enhancement of parametric pumping due to Andreev reflection. Appl. Phys. Lett. 79, 3977-3979 (2001).

24. Blaauboer, M. Charge pumping in mesoscopic systems coupled to a superconducting lead. Phys. Rev. B 65, 235318 (2002).

25. Taddei, F., Governale, M. \& Fazio, R. Andreev interference in adiabatic pumping. Phys. Rev. B 70, 052510 (2004).

26. Roddaro, S. et al. Hot-electron effects in InAs nanowire Josephson junctions. Nano Res. 4, 259-265 (2011).

27. Harris, R. E. Cosine and other terms in the Josephson tunneling current. Phys. Rev. B 10, 84-94 (1974)

28. Castro Neto, A. H., Guinea, F., Peres, N. M. R., Novoselov, K. S. \& Geim, A. K. The electronic properties of graphene. Rev. Mod. Phys. 81, 109-162 (2009).
29. Wei, Y. \& Wang, J. Carbon-nanotube-based quantum pump in the presence of a superconducting lead. Phys. Rev. B 66, 195419 (2002).

30. Jiang, X. et al. InAs/InP radial nanowire heterostructures as high electron mobility devices. Nano Lett. 7, 3214-3218 (2007).

\section{Acknowledgements}

We gratefully acknowledge M. Büttiker, L. Faoro, R. Fazio, L. B. Ioffe, J. König, A. F. Morpurgo, J. P. Pekola, V. Piazza, H. Pothier and S. Russo for discussions, and D. Ercolani for providing the InAs nanowires. The work was partially supported by the NanoSciERA project 'NanoFridge'. F.T. acknowledges financial support from the EU through the projects 'SOLID' and 'GEOMDISS'.

\section{Author contributions}

F.G. conceived and designed the experiment, and performed and analysed the measurements. P.S. performed and analysed the measurements. S.R. and S.B. fabricated the samples. F.T. and M.G. developed the theoretical model. L.S. set up the chemical beam epitaxy facility and contributed to the growth of InAs NWs. F.G., F.T. and M.G. wrote the manuscript with input from all coauthors.

\section{Additional information}

The authors declare no competing financial interests. Supplementary information accompanies this paper on www.nature.com/naturephysics. Reprints and permissions information is available online at http://www.nature.com/reprints. Correspondence and requests for materials should be addressed to F.G. 